\title{
Perspectivas da ética em pesquisa: o repensar para o futuro do sistema normatizador brasileiro
}

\section{Perspectives of research ethics: the rethinking for the future of the brazilian standardizer system}

\author{
1 Dartel Ferrari Lima dartelferrari07@gmail.com \\ 2 Lohran Anguera Lima
}

1 Graduado em Fisioterapia (1983), Mestrado em Engenharia Biomédica pela Universidade Tecnológica Federal do Paraná e Doutorado em Medicina Preventiva pela Faculdade de Medicina da Universidade de São Paulo. Especialista (Residência) em Fisioterapia Neurofuncional e Fisioterapia Ortopédica Traumatofuncional (COFFITO). Especialista Lato Sensu em Biologia do Esporte e Preparação Física. Tutor EaD. Coordenador do Comitê de Ética em Pesquisa CEP-Unioeste. Avaliador do Sistema BASis (MEC/INEP). Professor Permanente do Programa de Pós-Graduação em Educação em Ciências e Educação Matemática na Universidade Estadual do Oeste do Paraná, Brasil. Universidade Estadual do Oeste do Paraná.

2 Médico formado pela Universidade Estadual do Oeste do Paraná. Ortopedista e traumatologista pela Santa Casa de São Paulo (Pavilhão Fernandinho Simonsen). Integrou a equipe médica do campeonato mundial de surfe (WSL) no Rio de Janeiro (2012 e 2013). Membro da Sociedade Brasileira de Ortopedia e Traumatologia (SBOT), da North American Spine Society (NASS) e da AOSpine. Santa Casa de Misericórdia de São Paulo - Departamento de Ortopedia e Traumatologia.

\section{RESUMO}

Os comitês brasileiros de ética em pesquisa com humanos têm sido convidados a repensarem algumas de suas práticas. Este artigo apresenta como objetivo analisar alguns desafios para fortalecer a governança operacional do sistema CEP/CONEP que possibilitem avançar na operacionalização das demandas de seus membros, das instituições proponentes de pesquisa, dos pesquisadores e dos participantes de pesquisa. 0 método utilizado foi a pesquisa narrativa. 0 texto inicia com a normatização que orienta a análise ética de pesquisas com humanos. Em seguida, reflete sobre algumas barreiras que limitam a efetivação do controle da ética, realçando a necessidade da formação e treinamento continuado dos avaliadores, o diálogo entre os atores envolvidos na pesquisa, a ação do controle social e as assimetrias no sistema, encerrando com destaques sobre a responsabilidade ética das instituições promotoras de pesquisas com humanos. Conclui-se que a garantia da governança dentro de um sistema em expansão e a qualidade e a celeridade da análise dos projetos de pesquisa dependem de ações sincronizadas da capacitação continuada, do fortalecimento do diálogo entre os atores envolvidos no processo de pesquisa e na diminuição das assimetrias entres os CEP e entre membros de um mesmo CEP.

\section{Palavras-chave:}

Comitê de ética. Formação continuada. Pesquisa científica. Produção do conhecimento. Sistema CEP/CONEP.

\begin{abstract}
Brazilian human research ethics committees have been invited to rethink some of their practices. This article aims to analyze some challenges to strengthen the operational governance of the CEP/CONEP system in order to advance the operationalization of the demands of its members, the institutions proposing research, researchers, and research participants. The method used was narrative research. The text begins with the norms that guide the ethical analysis of research with humans. Then it reflects on some of the barriers that limit the effectiveness of ethics review, emphasizing the need for education and continuous training of reviewers, the dialogue among the actors involved in research, social control and asymmetries in the system, closing by giving emphasis on the ethical responsibility of the institutions promoters of human researches. It is concluded that the assurance of governance within an expanding system and the quality and speed of analysis of research projects depend on synchronized actions of continuous training, strengthening of the dialogue between the actors involved in the research process and the reduction of asymmetries between CEPs and between members of the same REC.
\end{abstract}

\section{Keywords:}

CEP/CONEP system. Continuing education. Ethics committee. Knowledge production. Scientific research.

\section{Como você deve citar?}

LIMA, Dartel Ferrari; LIMA, Lohran Anguera. Perspectivas da ética em pesquisa: o repensar para o futuro do sistema normatizador brasileiro. Cadernos UniFOA, Volta Redonda, n. 45, p. 89-95, abril 2021. 


\section{INTRODUÇÃO}

O respeito pela dignidade humana é um conceito central na ética em pesquisa. Implica o reconhecimento do valor intrínseco de todos os seres humanos, bem como a consideração que é devido a eles: o respeito pela integridade, a preocupação com o bem-estar e a participação justa e equitativa nas pesquisas (LYET; MOLLINA, 2018). Alguns indivíduos ou comunidades enfrentam um risco maior que o usual para participação em pesquisas. Esses riscos podem surgir de uma variedade de fontes, como a imaturidade, a vulnerabilidade e o comprometimento cognitivo. A pobreza e a falta de acesso a cuidados de saúde também podem tornar os indivíduos vulneráveis à coerção, exploração ou outros riscos. Essas condições, na investigação científica, exigem um pensamento renovado sobre o controle ético na pesquisa (BLANCHET, 2009).

Como primeiro passo, deve-se perguntar se os Comitês de Ética em Pesquisa no Brasil estão habilitados para atender aos requisitos do ambiente científico em vigor. Numa segunda etapa, não se pode evitar questionar o significado da integridade científica que as instituições proponentes colocam em prática. $\mathrm{O}$ aumento constante de metas administrativas, o tempo escasso do pesquisador por excesso de atividades, os impasses financeiros, a pressão da concorrência e as mudanças de comportamento social podem incentivar os investigadores a usarem meios questionáveis ou impróprios para chamar a atenção para o seu trabalho. A nova economia do conhecimento e seus requisitos representam grandes riscos para a ciência. Desse modo, é papel da academia trazer à tona uma reflexão ética, estabelecendo limites para proteger o investigado e dar credibilidade à atividade científica (LYET, 2018).

No contexto dessa reflexão, a palavra "ética" refere-se a todos os valores a respeitar no contexto de uma atividade de investigação envolvendo seres humanos, bem como as regras e os princípios que fluem deles; "pesquisa" é definida como a abordagem para o desenvolvimento do conhecimento mediante estudo estruturado ou investigação sistemática (LIMA, 2009; MALACARNE et al., 2011).

A partir do conceito firmado sobre ética em pesquisa, o seu controle não se define em um conjunto específico de regras ou normas. Em vez disso, fornece uma estrutura para avaliar problemas e determinar um curso de ações apropriadas. Desse modo, a análise ética deve refletir tanto as normas internacionalmente aceitas quanto o zelo de valores culturais e de costumes das comunidades onde a pesquisa é aplicada (SANTOS, 2018).

\section{O SISTEMA CEP/CONEP}

No Brasil, a Comissão Nacional de Ética em Pesquisa (CONEP) é uma comissão vinculada ao Conselho Nacional de Saúde (CNS), criada por meio da Resolução 196/96, com a função de implementar as normas e diretrizes regulamentadoras de pesquisas envolvendo seres humanos, atuando conjuntamente com uma rede de Comitês de Ética em Pesquisa (CEP), organizado de modo descentralizado, coordenado e sistêmico nas instituições onde as pesquisas se realizam, com a função de revisar estudos propostos com envolvimento humano e garantir que eles estejam em conformidade com as normas internacional e localmente aceitas. A integração da CONEP e do CEP doravante é denominada sistema CEP/CONEP (BRASIL, 2017).

O sistema CEP/CONEP, se propõe, em muitos aspectos, a mudar tanto a preparação quanto a implementação de trabalho de pesquisa que envolve seres humanos. Com normas orientadoras de proteção aos participantes de pesquisa, os projetos passam por uma análise preliminar em um dos 826 comitês de ética do Brasil (até dezembro de 2018), o que de certo modo, tem produzido resultados positivos de proteção da dignidade humana e um grande número de questões não resolvidas 
(BINSFELD, 2018). Esse sistema, ainda em expansão - com cerca de 60 novos CEP ingressando a cada ano - defronta-se com barreiras que o desafia. A apresentação dessas barreiras pode ser uma forma privilegiada de repensar ou mesmo fortalecer coletivamente o sistema para um modo de governança mais operacional, efetivo e célere às submissões que o aferem e possibilitar, ao sistema, avançar na operacionalização de diferentes demandas dos membros dos CEP, das instituições proponentes de pesquisa, dos pesquisadores e dos participantes de pesquisa.

Atualmente (em 2021), o sistema CEP/CONEP tem na sua gênese a busca de ações coordenadas, descentralizadas e sistêmicas para formar uma rede de proteção aos participantes de pesquisa em todo o território nacional. Desse modo, o sistema CEP/CONEP é percebido como uma rede interligada por 851 ramificações (CEP) que alcançam os centros de pesquisas espalhados pelo Brasil, gerenciados por um núcleo normativo (CONEP).

Essa estrutura requer uma organização funcional capaz de atender a demanda anual de, aproximadamente, 14 mil membros de CEP (média nacional de 17 membros por CEP), 90 mil projetos tramitados e 72 mil novos projetos iniciados, 600 mil pesquisadores cadastrados na Plataforma Brasil (base nacional e unificada de registros eletrônicos de pesquisas envolvendo seres humanos para todo o sistema CEP/CONEP) e, aproximadamente, 3 milhões de participantes de pesquisa (BINSFELD, 2018).

A dimensão atual do sistema e o seu crescimento exponencial não permite outro entendimento que não seja o pensar e o agir sistêmico. A ação isolada dos CEPs, além de fragilizar o local, danifica potencialmente o sistema. 0 pensar e o agir de mais de 14 mil membros dos CEP deve produzir uma consonância entre a previsão normativa dos regulamentos legais e a prática existente na análise dos projetos de pesquisa. A assimetria das decisões, fortemente vinculadas às diferenças culturais e pessoais dos membros dos CEP, estabelece, não em ordem de importância, a primeira barreira a ser ultrapassada pelo sistema CEP/CONEP.

\section{OS DESAFIOS AO SISTEMA CEP/CONEP}

A ética não se concretiza por decreto nem por normas rígidas (LIMA; MALACARNE, 2009) e não deve se prender às medidas burocráticas e/ou cartoriais para dificultar a ação do pesquisador (LIMA, 2009). Desse modo, urge o diálogo para o enfrentamento das necessidades dos CEP. Os cursos de formação inicial e capacitação continuada constitui pauta obrigatória de ação aliada para harmonizar a tomada de decisões (SILVA; MACHADO, 2018). Como estratégia, não se pode desviar a necessidade de focar o treinamento em função do público-alvo, do nível e do objetivo de cada participante.

A abordagem aos CEPs deve considerar a heterogeneidade dos comitês e de seus membros. As assimetrias no sistema CEP/CONEP fizeram com que, em 2018 , cerca de $96 \%$ dos projetos aprovados pelos CEP apresentassem alguma inconsistência ética, quando reavaliados pela CONEP (LIMA, 2009). Desse modo, a assimetria nos CEP necessita constante monitoração pelo sistema e pode ser atenuada com a formação continuada. Também, analisando o tempo de permanência de cada membro no CEP Unioeste, não escapou a observação que há forte rotatividade entre eles e que se postula como um possível mecanismo desencadeador dessa rotatividade, o desinteresse pelo controle social das questões éticas envolvidas em pesquisas.

O sucesso da renovação dos membros de um CEP parece fortemente vinculado à necessidade de treinamento inicial e continuado. Desse modo, o primeiro passo na organização de um programa de treinamento em ética em pesquisa é decidir a abrangência. A ampliação das oportunidades baseia-se na elaboração de diferentes estratégias para atender diferentes CEPs e diferentes públicos (LIMA; MALACARNE, 2009). 
No Brasil, no ano de 2017, mais de 300 CEPs analisaram até 50 protocolos no ano (cerca de quatro protocolos por mês); outros analisaram ainda menos ( 15 protocolos no ano) e outros, muito mais (acima de 1.000 protocolos por ano). Não cabe aqui fazer juízo do que é bom ou ruim em questão de demanda, pois é importante ter a percepção sobre o uso de uma estratégia diferenciada de ação capaz de atender o grande e o pequeno e que uma única estratégia para todos parece não ser adequada (PESSANO et al., 2015).

De modo adicional, as propostas de programas de treinamento continuado podem oferecer cursos com sessões mais curtas, limitando-os à solução de problemas focais. A estratégia de ensino a distância (EaD) se apresenta como ferramenta adequada para os cursos de longa duração para exercerem as suas funções com controle racional e razoabilidade (LIMA et al., 2020).

Muito embora haja uma programação anunciada pela CONEP no sentido de se concentrar na capacitação continuadas dos CEPs, até o ano de 2018 a atuação do sistema CEP/CONEP não atendeu essa necessidade. Os escassos cursos oferecidos aos CEPs e demais atores do processo focaram nos princípios e regras dessa forma de ética, e essa maneira de realização parece limitada. As abordagens da ética na pesquisa não têm desempenhado o papel de mediação da busca de preencher a lacuna entre os ideais e os objetivos da pesquisa e as condições pelas quais os projetos de pesquisa podem gerar riscos para os participantes e para a sociedade.

Outro desafio intrigante ao sistema CEP/CONEP reporta aos pontos nebulosos da Resolução 510/2016, que dispõe sobre as normas aplicáveis às pesquisas em Ciências Humanas e Sociais. Por razões históricas, as questões éticas estão há tempo no centro das preocupações das ciências biomédicas. No Brasil, mais recentemente, as humanidades não escaparam dessa incursão da ética nas práticas de pesquisa e foram particularizadas, mas o equívoco de transferência das regras das ciências biomédicas para o campo das humanidades parece ainda não totalmente superado.

Parece persistente também a subordinação de membros de CEP à cultura tradicional de avaliar todos os projetos sob a óptica biomédica. Portanto, faz-se necessário o treinamento específico para que a nova Norma seja atendida à luz do conhecimento preciso das funções das ciências humanas e sociais, dos tipos de pesquisa que propõem e dos aspectos éticos peculiares desse campo de estudo, deixando a intuição biomédica de lado para dar lugar a uma métrica própria e não copiada, distinguindo os tipos de pesquisa e seus possíveis e prováveis efeitos.

Nesse contexto de ultrapassar barreiras, não menos importante é fortalecer o diálogo entre os atores envolvidos na pesquisa. Para enfrentar esse desafio, o sistema CEP/CONEP poderia estabelecer um canal direto de comunicação com a comunidade acadêmica. Essa comunicação permanente de pesquisadores e membros de CEP poderia fortalecer claramente o limite aceitável da linha de demarcação entre o bem e o dano potencial da pesquisa, desviando, assim, da monocultura da informação (NUNES, 2017).

Desde o estabelecimento do sistema CEP/CONEP, em 1996, o diálogo educacional parece quase ausente entre os atores envolvidas em pesquisa. Os CEPs e a CONEP pouco têm investido nas questões educacionais dos pesquisadores, ocupando-se exclusivamente na análise de projetos. A falta de diálogo, entre outros prejuízos, tem produzido grande quantidade de pendências dos protocolos de pesquisa. Essas pendências, por sua vez, resultam em morosidade da efetivação da pesquisa. Algumas vezes, a morosidade da análise ganha tamanha dimensão e inviabiliza a realização (SENTER; RAYMUNDO, 2018).

A ocorrência de pendências de projetos é o fator que mais afeta a celeridade da tramitação. No ano de 2017, o tempo médio de tramitação de um projeto, em um cenário ideal, (sem pendência), nas duas instâncias do sistema (CEP e CONEP), foi de 50,6 dias (23,6 dias no CEP e 27 dias na CONEP). Ao receber uma única pendência, muitas vezes, por inconsistências de menor complexidade, a média de 
dias de tramitação se elevou para 131,8 dias (23,6 dias, no CEP, e 50 dias, na CONEP) (BINSFELD, 2018). Portanto, a proposta de diálogo fácil e simples requer entendimento claro e objetivo do agir racional e efetivo para se consolidar o vínculo com a racionalidade e a razoabilidade ao equiparar uns aos outros, consolidando o pesquisador e o membro dos CEP a partir dos sujeitos que são e de como poderiam ser.

Outro desafio eminente é a modernização da Plataforma Brasil, base digital e unificada de registros de pesquisas, criada pelo Governo Federal, em 2012, com o apoio da secretaria de Secretaria de Ciência, Tecnologia e Insumos Estratégicos (SCTIE), para sistematizar o recebimento dos projetos de pesquisa que envolvam seres humanos nos CEP em todo o país, propiciando o acesso público aos dados de todas as pesquisas aprovadas. À medida que a demanda por dados cresce, as ferramentas das plataformas eletrônicas passam por problemas de desempenho de extração, carregamento e transformação, necessitando de processos e de processadores de melhor escalabilidade (BRASIL, 2018).

A Plataforma Brasil, inegavelmente, foi um passo importante para a transparência e agilidade no processo do controle social da eticidade na pesquisa, permitindo e entrada de cerca de 72 mil novos projetos e outros 90 mil projetos transitados por ano dentro de sistema, além da hospedar mais de 600 mil pesquisadores cadastrados e outros 14 mil membros de CEP. No entanto, a plataforma não foi idealizada para suportar tamanha demanda e se encontra no limite operacional (BINSFELD, 2018). Assim, apresenta dificuldades para lidar com o volume atual de dados, para lidar com cargas de trabalho extremas, para recuperar rapidamente material arquivado, para acessar rapidamente informações, especialmente os documentos dos projetos de pesquisa; não é responsivo funcionalmente para smartphones e se hospeda em uma página na website instável. A atualização da Plataforma Brasil, de responsabilidade federal, representa um conjunto de desafios de infraestrutura capaz de fornecer rapidez e segurança para a captura, armazenamento e processamento de ampla variedade de informações por diferentes fontes.

O reconhecimento do sistema CEP/CONEP consolida o seu funcionamento a partir de sua identidade e da participação harmônica dos atores envolvidos no processo de controle social da ética em pesquisas envolvendo seres humanos, seja na instância de articulação, pactuação ou deliberação, para garantir a sustentabilidade do sistema mediante a participação do controle social.

A ação comunitária desempenha um papel vital para o controle social, mas não suficiente para promover, sozinho, o controle ético nas pesquisas. A participação comunitária, prevista no sistema CEP/ CONEP, reserva $15 \%$ de vagas para membros usuários, ou seja, de cada sete membros do sistema, um deve ser ocupado por usuário, independentemente de seu grau de formação. Os Conselhos Municipais de Saúde têm atendido ao chamado dos CEP, no entanto a aderência desse usuário como membro do CEP é um desafio ainda sem solução. Não raro, há grande rotatividade de membros usuários dentro de um CEP ou, até mesmo, por falta de voluntariado, um mesmo usuário representa a categoria em diversos CEP ao mesmo tempo. Os motivos da rotatividade são variados, de ordem econômica, sociocultural ou pessoal. Desse modo, é necessário que o sistema garanta as condições mínimas de aderência desse representante, fornecendo-Ihe as condições financeiras de deslocamento para as reuniões, a participação em capacitação e treinamento e a criação de condições para o afastamento do trabalho sem prejuízos, acompanhando o modelo firmado aos Conselheiros de Saúde pela Resolução 453/2012 (BRASIL, 2012).

Embora as propostas apresentadas no curso do artigo sejam endereçadas ao componente federal do sistema CEP/CONEP, algumas delas podem ser implementadas no âmbito local com o apoio das instituições. A responsabilidade de proteger os participantes humanos da pesquisa é compartilhada e as instituições devem avaliar suas políticas, procedimentos e práticas para assegurar que a infraestrutura básica para supervisionar a pesquisa esteja em vigor, que cada parte tenha conhecimento sobre seu papel e que haja linhas de comunicação abertas entre todos os setores institucionais. 


\section{CONSIDERAÇÕES FINAIS}

A garantia da governança dentro de um sistema em expansão deve focar na qualidade e na celeridade da análise e da tramitação dos projetos de pesquisa. Esses dois processos são dependentes de ações sincronizadas da capacitação continuada, do fortalecimento do diálogo entre os atores envolvidos no processo de pesquisa e na diminuição das assimetrias entres os CEPs e entre membros de um mesmo CEP. Nessa perspectiva, se reforça a necessidade do diálogo entre os atores envolvidos na pesquisa, para que se possa minimizar o impacto das pendências no tempo de tramitação das propostas.

Parece não ser suficiente o anúncio do sistema CEP/CONEP sobre o desenvolvimento de padrões rigorosos que protejam o público contra os riscos potenciais de certos projetos de pesquisa. 0 sistema deve promover espaços de capacitação e reflexão interdisciplinar sobre a ética e as condições de atuação do pesquisador. Na mesma linha, as instituições que abrigam os pesquisadores e as pesquisas têm a responsabilidade de fortalecer os mecanismos de controle social pela participação dos cidadãos, propiciando um trabalho crítico que não seja conduzido apenas pelas autoridades reguladoras ou pelos comitês de ética. $O$ que se espera dos sistemas reguladores é que se possa avançar com segurança e efetividade, da ética da pesquisa para a ética em pesquisa.

\section{REFERÊNCIAS}

BINSFELD, P. C. Desafios atuais do sistema CEP/CONEP. Formação Continuada dos Comitês de Ética em Pesquisa do RS, SC, PR, Joinville, 2018.

BLANCHET, P. La réflexivité comme condition et comme objectif d'une recherche scientifique humaine et sociale. Dans Cahiers de sociolinguistique. Paris, v. 1, n. 14, p. 145 - 152, 2009. Disponível em: https:// www.cairn.info/revue-cahiers-de-sociolinguistique-2009-1-page-145.htm. Acesso em: 03 jan. 2019.

BRASIL. Ministério da Saúde. Conheça a Conep. Brasília (DF), 2017. Disponível em: http://bvsms.saude. gov.br/bvs/publicacoes/conheca_conep_comissao_nacional_etica.pdf. Acesso em: 18 jan. 2019.

BRASIL. Ministério Da Saúde. Manual de Usuário: Pesquisador. [Internet]. Disponível em: http://www4. pucsp.br/cometicasorocaba/download/Manual-de-pesquisador.pdf. Acesso em: 16 jan. 2019.

BRASIL. Resolução N.453 do CNS de 10 de maio de 2012. Institui as diretrizes para instituição, reformulação, reestruturação e funcionamento dos Conselhos de Saúde. Diário Oficial [da] República Federativa do Brasil, Brasília, DF, 6 jun. 2012. Seção 1, p. 138.

LIMA, D. F. A cumplicidade entre os comitês de ética em pesquisa em seres humanos e as ações investigatórias. Caderno de Educação Física, Marechal Cândido Rondon, v. 8, n. 14, p. 97-102, 2009. Disponível em: http://e-revista.unioeste.br/index.php/cadernoedfisica/article/view/2161/2063. Acesso em: 26 jan. 2019.

LIMA, D. F.; LIMA, L. A.; MALACARNE, V.; CRISTOFOLETTI, J. F. Ética em pesquisa e responsabilidades sociais dos pesquisadores: uma perspectiva do CEP Unioeste. Revista Varia Scientia - Ciências da Saúde, Cascavel, v. 11, n. 1, p. 56-62, 2020.

LIMA, D. F.; MALACARNE, V. Ética em pesquisa envolvendo seres humanos: reflexões a partir das experiências da UNIOESTE - ciência e educação. ETD - Educação Temática Digital, Campinas, 
v. 11, n. 2, p. 175-205, dez. 2009. Disponível em: http://www.brapci.inf.br/_repositorio/2010/01/ pdf_788088c219_0007777.pdf. Acesso em: 10 jan. 2019.

LYET, P.; MOLINA, Y. Épistémologie éthique dans un espace interprétatif partagé et négocié. Le cas d)une recherche conjointe québéco-française. Québec, v. 59, n. 1-2, p. 225-241, 2018. Disponível em: https://www.erudit.org/fr/revues/rs/2018-v59-n1-2-rs03975/1051432ar/. Acesso em: 03 jan. 2019.

MALACARNE, V.; STRIEDER, D. M. LIMA, D. F. Ética, ciência e formação de professores: a escola na sociedade contemporânea. Ens. Pesqui. Educ. Ciênc., Belo Horizonte, v. 13, n. 3, p. 51-66, 2011.

NUNES, F. G. Desafios da pós-graduação: articulação entre ensino, pesquisa e extensão e diálogo com outras formas de produção do conhecimento. Atos de Pesquisa em Educação, Blumenau, v. 12, n. 1, p. 23-35, 2017.

PESSANO, E. F. C.; LANES, K. G.; LANES, D. V. C.; FOLMER, V.; PUNTEL, R. L. A contextualização como estratégia para a formação continuada de professores em uma unidade de atendimento socioeducativo. Revista Electrónica de Enseñanza de las Ciencias, Vigo, v. 14, n. 3, p. 340-360, 2015. Disponível em: http://reec.uvigo.es/volumenes/volumen14/REEC_14_3_5_ex916.pdf. Acesso em: 15 jan. 2019.

SANTOS, C. A. S. Regulamentação ética das pesquisas no Brasil: precisamos de uma resolução especifica para Ciências Sociais e Humanas? Revista Iberoamericana de Bioética, Madrid, n. 06, p. 01 12, 2018. Disponível em: https://revistas.upcomillas.es/index.php/bioetica-revista-iberoamericana/ article/.../800. Acesso em: 04 jan. 2019.

SENTER, L.; RAYMUNDO, G. I. M. C. Desafios na Implantação de Cursos a Distância. Revista EaD em Foco, Rio de Janeiro, v. 8, n. 1, p. e703, 2018. Disponível em: eademfoco.cecierj.edu.br/index.php/Revista/ article/download/703/319. Acesso em: 14 jan. 2019.

SILVA, G. F.; MACHADO, J. A. Saberes em diálogo: a construção de um programa de formação docente em uma rede municipal de ensino. Revista Ibero-americana de Educação, Araraquara, v. 77, n. 2, p. 95 114, 2018. Disponível em: https://rieoei.org/RIE/article/download/3161/3978/. Acesso em: 14 jan. 2019. 